\section{Cahiers de Narratologie}

Analyse et théorie narratives

$35 \mid 2019$

Le style comme événement

\title{
La valeur romanesque de la liste. Entretien avec Gaspard Turin
}

Propos recueillis par Raphaël Baroni

\section{Raphaël Baroni}

\section{OpenEdition}

\section{Journals}

Electronic version

URL: http://journals.openedition.org/narratologie/9490

DOI: 10.4000/narratologie.9490

ISSN: 1765-307X

\section{Publisher}

LIRCES

\section{Electronic reference}

Raphaël Baroni, « La valeur romanesque de la liste. Entretien avec Gaspard Turin », Cahiers de

Narratologie [Online], 35 | 2019, Online since 03 September 2019, connection on 15 November 2019

URL : http://journals.openedition.org/narratologie/9490; DOI : 10.4000/narratologie.9490

This text was automatically generated on 15 November 2019.

Article L.111-1 du Code de la propriété intellectuelle. 


\title{
La valeur romanesque de la liste. Entretien avec Gaspard Turin
}

\author{
Propos recueillis par Raphaël Baroni
}

\section{Raphaël Baroni}

1 Cet entretien revient sur le dernier ouvrage de Gaspard Turin, Poétique et usages de la liste littéraire. Le Clézio, Modiano, Perec, publié chez Droz en 2017. Dans ce livre, préfacé par Philippe Hamon, Gaspard Turin présente la liste comme «l'un des symptômes scripturaux les plus prégnants de notre époque » et il se propose d'en décrire les usages romanesques par plusieurs écrivains français récents. Il montre que cette forme oscille entre ordre et chaos, entre l'hybris d'un rêve de saisie totale du monde et la mélancolie d'une tentative vaine de retenir ce qui est voué à l'oubli. Surtout, l'auteur démontre la fécondité d'une approche orientée sur la liste pour saisir le paysage littéraire moderne et contemporain et pour comprendre ses enjeux profonds. À la fois très dense d'un point de vue théorique et riche dans ses analyses adossées aux œuvres de Le Clézio, Modiano et Perec, ce livre s'annonce comme un jalon essentiel autant pour la poétique contemporaine que pour une critique soucieuse d'ancrer la compréhension de l'usage des listes dans un contexte biographique et historique.

Raphaël Baroni : Pour commencer cet entretien, j'aimerais vous demander de revenir brièvement sur la manière dont la liste s'est imposée à vous comme un objet d'investigation. Comment le désir de mieux comprendre ce phénomène vous est-il venu dans votre parcours de chercheur?

Gaspard Turin: Il s'agit, comme souvent, d'une conjonction un peu hasardeuse d'évènements. D'abord un intérêt, à l'époque de mes études, pour l'esthétique du fragment et le souhait d'interroger ce qui se présente à mes yeux comme l'objet textuel le plus radical de cette esthétique. Il me semblait, et il me semble toujours, que dans le domaine de la littérature comtemporaine l'histoire et la poétique des formes était déterminante, précisément à cause d'un divorce progressif de la littérature d'avec les formalismes de l'après-guerre. Puis je faisais le constat que la liste pouvait correspondre, contre toute attente, à un travail académique d'ampleur - d'autres s'y mettaient, U. Eco, B. Sève, M. Jeay - avec ce que cela comporte de sérieux mais aussi de 
jubilatoire. Se dévoilait aussi, à travers eux, l'extraordinaire fortune de cette forme de liste dans le corpus littéraire, la cornucopia au principe d'œuvres historiquement, géographiquement et génériquement des plus diverses, de Homère à Auster, de Villon à Whitman ou à Gomez de la Serna. En parallèle, je faisais la découverte progressive d'œuvres contemporaines comme celles d'Annie Ernaux, de Pascal Quignard, d'Éric Chevillard, d'Antoine Volodine, de Pierre Senges, d'Olivia Rosenthal... Au fil du temps et des lectures, un fantastique vertige babélien vous prend, lorsque vous constatez que ces auteurs « à liste » font eux-mêmes liste, et que cette juxtaposition ou cette asyndète des auteurs nous livre une partie de leur ethos, de leur présence dans le champ littéraire, selon une éthique de la multitude chère à Yves Citton et à Lionel Ruffel.

$\mathrm{RB}$ : La première partie de votre ouvrage propose une conceptualisation de ce que vous définissez comme une "figure-limite». Est-ce que vous pourriez revenir rapidement sur cette définition et sur les raisons qui font de cette figure un objet aussi difficile à saisir.

GT : Le problème de la définition de la liste tient justement dans sa forme même, qui s'oppose à la plupart des modes définitoires envisagés par la théorie littéraire lorsqu'elle cherche à comprendre un objet. La liste pure, si un tel objet existe - ce que je ne crois pas, mais qu'il faut admettre heuristiquement - est non-apophantique, pour reprendre ce terme d'Aristote; elle se refuse à faire fonctionner de manière simple le caractère affirmatif de la prédication. Elle s'oppose à toute définition essentialisante, qui sert de modèle aux dictionnaires et aux encyclopédies. Elle ne dit jamais « A est $\mathrm{P}$ ", elle dit toujours « $A$ est $P, Q, R, S, T$ » et dès lors lutte contre le caractère assignatif de la définition. Et cette qualité particulière se déporte sur les propriétés mêmes de la liste, qui non seulement sont très diverses, mais également oppositionnelles. Ainsi une liste peut se révéler, comme on le sait, ouverte ou fermée ; mais selon qu'elle est l'un ou l'autre, elle ne recoupera plus la même réalité praxéologique : sa pratique, ses usages seront incompatibles.

Dès lors, les tentatives de définir l'objet liste ne peuvent englober tout ce qu'il peut être, mais comme il faut bien de temps en temps refermer la conceptualisation, je considère i. qu'on ne peut nommer « liste » qu'un objet verbal et discursif, à l'exclusion de l'image par exemple, et ii. qu'elle se reconnaît à la commensuration des items qui la composent, c'est-à-dire à la tendance plus ou moins marquée de ses items à se ressembler morphologiquement et à se différencier sémantiquement. De cette conceptualisation émane la centralité de l'asyndète énumérative, qui constitue la figure fondamentale de la liste, en français du moins. Or il faut tout de suite observer que l'asyndète est une figure particulière, en ce qu'elle est soustractive : c'est la suppression des termes conjonctifs d'un énoncé. C'est, en fait, la mise en évidence des blancs entre les items. L'asyndète, comme elle n'ajoute rien mais retranche, laisse en quelque sorte la liste à elle-même, à sa propre économie minimale. La raison pour laquelle je considère la liste comme une figure-limite tient à cet isolement, puisque la liste ne concourt pas à la construction ordinaire de la phrase ou de l'énoncé, mais s'y oppose, à son propre bénéfice et à celui, éventuellement, de son énonciateur.

7 RB : La partie théorique de votre ouvrage n'insiste pas seulement sur la "poétique » de la liste, mais également sur ce que vous définissez comme une «éthique ", qui associe cette figure à une sorte de balancement entre deux pôles définis par leur tonalité « thymique » : l'hybris et la mélancolie. Pourriez-vous définir ces deux formes extrêmes et expliquer comment cette polarité s'est dégagée de vos analyses. 
GT : À reprendre les catégories d'ouverture et de fermeture, je considère que la liste fermée n'est pas très intéressante pour la littérature. Je pense d'ailleurs qu'elle n'y existe pas. Son territoire est celui des productions écrites non immédiatement littéraires, comme le menu, le répertoire téléphonique, la recette, etc. Dans ces cas-là, l'item de liste est subordonné à son référent, son objet. Dans le cas de la liste ouverte, c'est avant tout son sujet qui compte, en l'espèce son énonciateur, initialement l'auteur ou sa fonction, mais l'énonciateur, par un effet de métalepse spécifique à la liste, se déporte aussi sur le lecteur qui se l'approprie à plusieurs égards. C'est à lui que se pose en dernière instance le choix de recevoir ou non la liste, le cas échéant de choisir comment la lire, puis de s'interroger sur l'opportunité de la prolonger par la sienne propre (car tout le monde a sa, ou ses listes, et le geste d'ajout coûte peu). Devant une liste littéraire, ce mouvement de praxis métaleptique trouve deux issues qui se caractérisent par une attitude thymique, dérivée de l'euphorie ou de la dysphorie, que j'appelle respectivement hybris et mélancolie. L'hybris consiste à croire que la nomination répétée, et potentiellement infinie, des objets du monde se confond avec le sentiment de toute-puissance, de maîtrise démesurée que l'on a sur eux, et sur le monde. C'est l'attention au plein de l'item. La mélancolie est le penchant inverse, consistant à réaliser que l'item à venir sera toujours celui qui manque.

RB : Avez-vous déjà envisagé l'existence d'autres prototypes pour la liste, par exemple celui, ludique, qui naîtrait d'une combinatoire au sein d'une liste fermée d'entités ? Estce que certaines œuvres oulipiennes, comme Le Château des destins croisés d'Italo Calvino, ou un roman comme Composition no. 1 de Marc Saporta, pourraient relever selon vous d'une logique apparentée à la liste?

10 GT : Oui - mais là aussi, il faut différencier liste littéraire et liste non-littéraire, ou pratique, et les différencier dans leurs usages plutôt que dans leur(s) forme(s). Maintenir la genèse d'une œuvre littéraire dans un cadre contraignant n'a jamais comme équivalent la fermeture d'une telle œuvre, alors qu'un tableau simple comme un doodle, par exemple, ne peut se satisfaire d'un reste qui ne soit pas dûment compris dans ses propositions initiales (les deux listes en abscisse et en ordonnée). Vous pouvez être disponible pour un événement, ne pas l'être, voire l'être éventuellement aux dates proposées, mais toute information supplémentaire n'a pas lieu d'apparaître à ce stade de l'organisation. Calvino, Cortázar, Perec savaient bien que l'écriture sous contrainte ne pose initialement le cadre que pour mieux le questionner, voire le briser en cours de route. Il me semble donc nécessaire de ne pas apparier trop radicalement le tableau à la liste (comme le fait l'anthropologue Jack Goody pour les besoins de sa démonstration), de ne pas faire systématiquement de celui-ci l'instrument de limitation de celle-là, qui structurellement ne contient pas le principe de sa propre finalité et peut donc se prolonger à l'infini.

11 RB : Vous avez aussi consacré plusieurs travaux à d'autres auteurs, comme Pascal Quignard. Pourrez-vous rapidement évoquer la place que tient la liste dans l'œuvre de ce dernier et la raison pour laquelle il n'est pas évoqué de manière plus centrale dans votre livre?

12 GT : L'intérêt que Quignard porte à la liste est lié à celui qu'il porte, depuis très longtemps, au fragment comme procédé d'écriture. En cela, je considère la liste chez Quignard comme le devenir ultime du fragment, et de nombreux passages de cette expédition pélagique dans la haute pensée et dans la haute langue qu'est Dernier royaume montrent cette tentation: extrême brièveté du propos, réduction des 
propositions syntaxiques, mise en péril de l'écriture jusqu'à toucher ses rivages antipodes, de l'aphasie, du silence. Antoine Volodine procède aussi, à sa manière, à de tels questionnements, et chez lui aussi la liste est un procédé esthétique et poétique central. Et on pourrait dire à peu près la même chose de l'œuvre de Patrick Modiano, de l'Annie Ernaux des Années... Il y en aurait d'autres. Il se trouve que, pour mon livre, qui est avant tout théorique, multiplier les exemples aurait eu pour effet d'en augmenter la taille de manière exponentielle. Les auteurs traités dans le livre (Le Clézio, Modiano, Perec) le sont d'ailleurs de manière lapidaire, je ne rends pas justice à la densité et à la richesse de leurs œuvres.

RB : En introduction, vous soulignez que la liste apparaît comme l'une des premières formes d'organisation textuelle historiquement attestée. En même temps, vous montrez que les formes romanesques contemporaines manifestent un intérêt particulièrement marqué pour elle. Pouvez-vous nous expliquer en quoi le champ littéraire français récent offre un terreau fertile pour analyser cette figure? Est-ce parce que la littérature d'aujourd'hui s'organise autour d'autres structures que celles découlant d'une approche strictement narrative?

GT : En premier lieu, il faut rappeler que ce "champ littéraire ", appréhendé sous l'angle sociologique que l'expression induit, se présente lui-même sous la forme d'une liste, par le caractère pléthorique du nombre de livres publiés par an, nombre qui semble n'avoir aucune limite. À ce point de submersion du quantitatif sur le qualitatif, le spécialiste de littérature contemporaine est bien obligé d'admettre qu'il ne peut lire, au mieux, que la liste de ces titres, au détriment des ouvrages eux-mêmes. C'est d'ailleurs la leçon principale de Franco Moretti et de son distant reading, à l'aune du traitement informatique auquel il soumet les textes sur lesquels il travaille: non seulement il ne s'agit pas de lire cinq mille romans (on ne peut pas), mais encore pour s'en faire une idée globale est-il nécessaire de ne pas les lire (il faut ne pas).

15 En second lieu, la situation éditoriale actuelle, pas seulement française, conduit à visibiliser parmi les centaines et centaines de romans (pour ne parler que d'eux) publiés chaque année un très petit nombre d'entre eux, généralement oublieux des possibilités formelles de la langue et de la fiction, des possibilités qu'offrent la dissidence générique, l'exploitation de genres paralittéraires, l'expérimentation.

Il me semble que la part la plus intéressante de la littérature contemporaine est justement composée d'auteure's qui comprennent que leur situation sociologique et leur situation esthétique sont liées. Leur « faire-liste » dans le champ littéraire peut, ou doit, s'accompagner d'une sorte de pas de côté, voire d'un refus frontal des systèmes exploités par les très gros vendeurs sur-visibilisés. Ceux-là exploitent généralement avant tout l'aspect scénaristique, le pré-mâchage cinématographique de leurs livres, accompagnent le spectaculaire médiatique et lui répondent, en bonne entente. Ce n'est à mes yeux pas un hasard de constater que de très nombreux auteurs, situés souvent sous la ligne de flottaison de la critique journalistique, exploitent aujourd'hui une veine thématique du hors-champ, du non-médiatique, du quotidien, du détail et que cet intérêt passe par le discontinu, la forme brève, la liste : Xavier Person, Éric Chevillard, Michèle Audin, Marcel Cohen, Yves Pagès, Olivia Rosenthal, Jérôme Game, Anne Savelli, Sophie Coiffier, Michelle Grangaud, PNA Handschin, Édouard Levé, entre autres (car la liste se perd dans l'infra-visible...)

17 RB : Pour renchérir sur ce point, vous mentionnez que la liste «dans sa structure, s'oppose au texte narratif» (p. 6). Pourriez-vous préciser cette opposition 
fondamentale entre la logique sérielle et a priori non bornée de la liste et celle présidant à la formation d'une intrigue, qui joue davantage sur la discontinuité, la téléologie d'une progression orientée vers un dénouement et une hiérarchisation fondée sur l'idée qu'il y aurait des moments forts et des moments faibles ? Est-ce qu'en filant une métaphore musicale, on ne trouve pas dans cette opposition quelque chose comme la différence qu'il y aurait entre une phrase musicale, par exemple une progression mélodique orientée vers une résolution, et une pulsation rythmique plus fondamentale, plus archaïque, organisée sur une forme de répétition binaire? Est-ce que liste et récit sont nécessairement dans un rapport d'opposition ou de tension, ou peuvent-il s'emboîter harmonieusement?

GT : Il est impossible de prétendre, surtout face à un spécialiste de l'intrigue, que le texte narratif ordinaire (essentiellement prédicatif) se contenterait d'une linéarité dans la progression de l'information. Mais je choisirais plutôt d'interroger, pour faire valoir tout de même une opposition à un texte narratif canonique supposé, et présentant cette qualité musicale mélodique dont vous parlez, la notion de disharmonie. Par exemple, Philippe Hamon qualifie de « kyste » la liste au sein du roman moderne, parce qu'il la considère comme à la limite de l'assimilable, d'ailleurs souvent rejetée, sautée par la lecture. Sans même aller jusqu'à ce point de refus, une écriture romanesque foncièrement digressive comme celles de Chevillard ou de Senges, et dont les digressions se spiralisent souvent dans la liste, jouent plutôt d'un inattendu, d'un changement de milieu. Changement qui, comme on le sait après Deleuze, Benveniste ou Meschonnic, a tout à voir avec le rythme, mais très peu avec la cadence. Je dirais donc, pour en rester dans la métaphore musicale, que les auteurs chez qui l'esthétique de liste est la plus fondamentale se rapprocheraient plus de la musique stochastique de Iannis Xenakis ou, dans un autre genre, du chaos organisé et virtuose de Frank Zappa ou de Mike Patton. Je ne pense pas que la musique électronique répétitive participe de l'esthétique de liste, parce que la répétition ne se confond pas avec la liste (qui est pour moitié répétition morphologique, pour moitié différence sémantique). A contrario, un roman comme Extinction de Thomas Bernhard, extrêmement répétitif mais dépourvu de listes, pourrait être qualifié de roman-techno.

19 RB : D'un point de vue comparatiste, pensez-vous que la littérature française présente un terreau plus fertile pour une prolifération fictionnelle de la liste que d'autres littératures, par exemple anglo-saxonnes ou germaniques?

GT : Probablement pas - seule ma relative méconnaissance des littératures étrangères m'empêche d'affirmer avec certitude que la liste est un objet littéraire universel, mais sa présence chez des auteurs aussi éloignés dans les langues, le temps et l'espace que Cervantes et Saint John Perse, Rabelais et Borges, Zola et Pynchon tend à faire admettre cette idée. Il faudrait sans doute en passer par des termes de grammatologie comparée pour différencier les pratiques canoniques de l'énumération entre les langues; par exemple, le français construit son énumération canonique par une asyndète bornée par un et avant le dernier item, et admet difficilement la polysyndète, hormis dans la négation (listes en $\mathrm{ni}$ ) ou l'alternation (listes en ou), tandis que l'anglais semble accepter plus facilement la polysyndète en and : "Your love says, like an honest gentleman, and a courteous, and a kind, and a handsome, and, I warrant, a virtuous-where is your mother? » (Shakespeare)... Mais il faudrait vérifier, je n'en suis pas certain. la littérature française moderne, mais vous montrez aussi qu'il émerge dans un 
contexte de prolifération de travaux académiques consacrés à cette forme littéraire. Pourriez-vous expliquer comment ce champ de recherche a pu se constituer et la manière dont votre travail se situe par rapport à d'autres approches apparentées ?

GT : L'intérêt actuel pour la liste est évidemment lié à un contexte socioculturel contemporain de pléthore et de crise de l'information. On constate de manière diffuse que les formes brèves remplacent les formes longues - même si un tel constat est difficile à pointer plus précisément, mais on peut admettre que, mathématiquement, s'il y a plus de choses à dire, il y a également moins d'espace et moins de temps pour les dire. Dans un tel contexte, on remarque que les ouvrages consacrés à la liste ou qui s'y intéressent sont souvent eux-mêmes des listes de listes; on retrouve, en régime hypermoderne, de vieilles habitudes d'appropriation du savoir datant de la Renaissance, d'une époque où se constituait l'individu moderne via, notamment, les premières collections, les cabinets de curiosité. Dans son rapport à la liste, le livre d'Umberto Eco Vertige de la liste (2009) n'est pas très éloigné de Au bonheur des listes de Shawn Usher (2014), lui-même tributaire des succès de librairie que furent La première gorgée de bière (1997) de Philippe Delerm ou Les miscellanées de Mr. Schott (2005) de Ben Schott.

Le danger, pour la recherche, de cette tendance, que l'on retrouve jusque dans l'ouvrage - même s'il est beaucoup plus rigoureux et fouillé - de Bernard Sève, De haut en bas. Philosophie des listes (2010), c'est le remplacement du discours articulé par le discours juxtapositionnel. C'est, en quelque sorte, la contagion de l'objet sur le sujet, qu'il ne faut pas prendre à la légère - et tout théoricien de la liste en est lui-même un usager, n'échappe pas à cette maladie. Pour ma part, j'ai cherché à éviter ce danger en proposant une théorisation préalable au corpus, ce qui présente un autre type de danger, parce qu'une structure hypotético-déductive comme la mienne tend à réduire par la suite son corpus à une exemplification du système exposé en amont. Mais j'ai aussi cherché à dépasser cette fatalité, en choisissant, en guise de corpus, un premier exemple illustrant le pôle de l'hybris (Le Clézio), un deuxième illustrant celui de la mélancolie (Modiano) et un troisième (Perec) chez qui les usages littéraires de la liste sont trop riches pour se satisfaire d'une telle structure dialectique.

RB : Pourriez-vous évoquer dans quelles directions futures vos recherches sur la liste vont se développer? En particulier, est-ce que vous voyez dans les humanités numériques, qui font massivement appel à des approches quantitatives fondées sur des listes, un lieu où votre réflexion critique pourrait apporter un angle critique inédit?

GT : Oui, c'est effectivement une piste de recherche qui m'intéresse au plus haut point. En particulier à un niveau anthropologique. Jack Goody, dans La raison graphique (1977) avait montré que l'apparition, il y a 4500 ans, de l'écriture au sein des sociétés mésopotamiennes (dont la complexité croissante exigeait la création d'une sorte de mémoire externe, qui s'est matérialisée par l'écrit), avait déterminé un changement de paradigme cognitif, et que toute la pensée occidentale était tributaire de l'organisation de la pensée par l'écrit - la littéracie, pour le dire rapidement. Dans cette révolution épistémologique, la liste jouait un rôle de premier plan; elle représentait l'isolement d'unités de langage auparavant perçues uniquement, selon Goody, dans le flux d'énonciations momentanées. La liste est le premier objet verbal qui mette en évidence la séparation des éléments qui le composent, ce qui historiquement a permis, via sa mise par écrit, la manipulation de ses items, leur déplacement hors de leur contexte 
initial d'énonciation, le jeu avec le langage, jusqu'à l'invention de la littérature dans le long moment moderne où celle-ci aura été indissociable de sa forme écrite.

Or il me semble, et les recherches d'un Moretti que j'ai mentionné plus tôt en sont un témoignage jusque dans la recherche, que dans son rapport au littéraire, l'individu contemporain est de plus en plus confronté à des listes et de moins en moins capable de traiter les formes écrites plus complexes. Que l'on ajoute à cela l'habitus technologique intégré aujourd'hui massivement par chacun: il n'en faut pas beaucoup plus pour observer que ce qui se joue ici serait l'usurpation de la littéracie par la computationnalité. Mais la question que sous-tend ce constat est profondément ébranlante : il ne s'agit de rien de moins que d'entériner la disparition des Lumières (comme le fait David M. Berry), voire de la pensée humaine moderne (comme le suggère Jussi Parikka). Le syntagme même d'humanités numériques s'inscrit en faux contre ce discours crépusculaire, et je ne sais pas encore, à titre personnel, s'il faut ajouter une trompette au siège de Jéricho ou chercher à consolider ses murailles...

RB : Comme l'indique votre introduction, la liste apparait comme une forme omniprésente dans notre culture, de la liste des courses à ce genre journalistique qui consiste à dresser des classements des objets culturels. J'aimerais terminer en élargissant un peu la perspective au-delà de la littérature. Comme je sais que vous manifestez également un intérêt marqué pour la critique musicale, qui vous a longtemps occupé avec le webzine Sonotone (http://sonotone.ch), pourriez-vous nous dire, en conclusion de cet entretien, les liens que vous voyez entre ces deux passions. Est-ce que la liste constitue le chaînon manquant entre vos différentes facettes identitaires? Et si vous le voulez bien, pourriez-vous nous livrer votre « top 5 » des plus grands albums et des plus grands romans du $\mathrm{XX}^{\mathrm{e}}$ siècle et les éventuels liens qui peuvent exister entre ces panthéons?

GT : Mon top 5 dans ces deux domaines ne reflètera pas uniquement mon travail sur la liste (qui ne m'obsède pas, contrairement à ce qu'on pourrait croire). Je me bornerai à citer un musicien chez qui la liste est centrale : Neil Hannon, à travers son projet The Divine Comedy, qui ne cesse depuis 25 ans de produire des chansons magnifiques, petits bijoux extrêmement polis et ajourés de pop orchestrale, dans la lignée du McCartney d' Eleanor Rigby et de Penny Lane, celle des Kinks et des Zombies. Le nom du projet de Hannon dit assez bien ses ambitions littéraires, à mon avis très justifiées, et les chansons à listes sont légion chez lui. L'une d'elles, The Booklovers, est uniquement constituée d'une liste de noms d'écrivains, suivis d'un gimmick amusant qui les résume (par exemple: «Herman Melville - Ahoy there!»). La démarche artistique est plutôt conservatrice et entre en plein dans le renouvellement de l'esprit de cabinet de curiosités que j'évoquais plus haut. Ce qui ne m'empêche pas de vénérer le travail de Hannon, que je glisse dans mon top 5 avec son deuxième album, Promenade (1994), indépassé à mes yeux, malgré une production faiblarde et d'autres excellents disques depuis.

Le livre qui entre dans mon top 5 et qui recoupe mon intérêt pour la liste est sans conteste La Vie mode d'emploi de Perec, livre majeur du XXe siècle au même titre que la Recherche, Ulysse, Le Procès, Le Bruit et la fureur ou Voyage au bout de la nuit, mais dont les résonances se sont manifestées dans la chambre d'écho des perecquiens eux-mêmes plus qu'en dehors. C'est un livre-monde, mais étrangement modeste. Il fait partie des rares livres qui creusent dans la bibliothèque une place plus importante que celle 
qu'occupe leur volume. C'est aussi le cas des romans de Chevillard, qui dit écrire « pour occuper moins de place ».

Les autres livres et disques que je rangerai dans les huit cases qui me restent ne sont pas forcément liés à mon travail sur la liste. Je les livre ici sans ordre particulier, ni commentaire, parce que nous en aurions pour trente pages supplémentaires. Me reste à préciser que la sélection dépasse le $\mathrm{XX}^{\mathrm{e}}$ siècle, parce que mes auteurs comme mes musiciens de prédilection apparaissent plutôt entre l'après-guerre et aujourd'hui. Il va de soi que je ne me ferai pas prier pour une discussion plus développée des mérites comparés de ces livres ou de ces disques, autour d'un whisky sour ou deux.

Marie NDiaye Rosie Carpe (2001)

Antoine Volodine, Des anges mineurs (1999)

Vladimir Sorokine, La tourmente (2011)

Jean-Patrick Manchette, Le petit bleu de la côte ouest (1979)

The Smiths, Meat is Murder (1985)

36

The Magnetic Fields, 69 Love Songs (1999)

37 Talking Heads, Fear Of Music (1979)

38 Stevie Wonder, Innervisions (1973) 